\title{
First Demonstration on Direct Laser Fabrication of Lunar Regolith Parts
}

Vamsi Krishna Balla ${ }^{1}$, Luke B. Roberson ${ }^{2}$, Gregory W. O’Connor ${ }^{3}$, Stephen Trigwell ${ }^{4}$, Susmita Bose $^{1}$ and Amit Bandyopadhyay ${ }^{1 *}$

${ }^{1}$ W. M. Keck Biomedical Materials Research Laboratory, School of Mechanical and Materials Engineering, Washington State University, Pullman, WA 99164, USA.

${ }^{2}$ National Aeronautics and Space Administration, Kennedy Space Center, FL 32899, USA.

${ }^{3}$ Amalgam Industries, Inc., 925 Estates Drive, Los Alamos, NM 87544

${ }^{4}$ ASRC Aerospace Corporation, Kennedy Space Center, FL 32899

$\left[{ }^{*}\right]$ Corresponding-Author: Prof. Amit Bandyopadhyay

Tel: 509-335-4862; E-mail: amitband@wsu.edu 


\begin{abstract}
Establishment of a lunar or Martian outpost necessitates the development of methods to utilize in situ mineral resources for various construction and resource extraction applications. Fabrication technologies are critical for habitat structure development, as well as repair and replacement of tools and parts at the outpost. Herein we report the direct fabrication of lunar regolith simulant parts, in freeform environment, using lasers. We show that raw lunar regolith can be processed at laser energy levels as a low as $2.12 \mathrm{~J} \mathrm{~mm}^{-2}$ resulting in nanocrystalline and/or amorphous microstructures. Potential applications of laser based fabrication technologies to make useful regolith parts for various applications including load bearing composite structures, radiation shielding, and solar cell substrates is described.
\end{abstract}

\title{
Introduction
}

Learning how to utilize in situ resources on the Moon is very critical to expanding human presence to Mars and across the solar system. Effective in situ lunar resource utilization for structures, materials, power generation, and other developments will reduce logistics and extend mission durations, and thus increase mission efficiencies as well as science return. Lunar regolith contains important materials that can be used for in situ resource utilization (ISRU) on the Moon, thereby providing for substantial economic savings for the development of a permanently staffed outpost. The Exploration Systems Architecture Study ${ }^{1}$ (ESAS) identified processing of raw regolith on the Moon as a critical area of ISRU that would provide significant benefits to future robotic and human exploration to the Moon. Molten oxide electrolysis process has been proposed to process raw regolith to produce oxygen for life support, silicon for solar cells, reactive metals for advanced storage batteries, and steel and aluminum for construction ${ }^{2,3}$. Alternatively, some studies have been performed to develop methods to use lunar regolith as a construction ${ }^{4-7}$, electronic substrate ${ }^{8}$, radiation shielding ${ }^{9,10}$, refractory ${ }^{11}$ and heat shield ${ }^{11}$ material. In situ Fabrication and Repair (ISFR) using locally provisioned and/or locally refined materials on the Moon require state-of-the-art fabrication technologies to support habitat structure, mechanical parts fabrication, and repair and replacement of ground support and space mission hardware (such as life support items and launch vehicle components). The processing or fabrication 
methods used so far in the development of useful products from lunar regolith require extensive tooling and the cost of transporting them from Earth to the Moon would be prohibitively expensive. Moreover, fabrication of construction elements ${ }^{4-6,10}$, tools/parts ${ }^{7}$ and electronic substrates $^{8}$ from lunar regolith via conventional processing such as melting/sintering would be highly energy intensive. Therefore, the fabrication hardware that does launch to the Moon must be mass and energy efficient.

Advanced manufacturing routes based on additive manufacturing (AM) technologies can be implemented to fabricate net shape parts, tools and other necessary items using in situ materials. Fundamentally, AM technologies produce complex-shaped three-dimensional (3D) objects directly from computer-aided design (CAD) models by successive addition of material(s) in layer-by-layer fashion without the use of specialized tooling, molds, or dies. The result is a net shaped part ready for cleaning and minimal post-fabrication finishing. Therefore, the application of AM technologies can significantly benefit ISFR on the Moon. Some studies ${ }^{12,13}$ evaluated the capabilities of AM technologies to produce metal based parts/components for ISFR. The metal feedstock such aluminum, iron, and titanium can be produced via Molten oxide electrolysis of lunar regolith ${ }^{2}$. However using raw regolith without any beneficiation as fabrication feedstock could be more appropriate/economical. In addition, due to the absence of hydrolytic weakening processes on the moon and in the hard vacuum of free space, lunar materials and their derivatives such as lunar silicate materials may possess very high geomechanical strengths compared to equivalent materials on earth. Thus, possible substitution of lunar silicate materials for structural metals in a variety of space engineering applications enhances the economic utilization of the moon ${ }^{14-16}$. Such substitution or direct use of raw regolith is not without its technological challenges. Herein we report successful fabrication of bulk, near net shape, lunar regolith simulant parts using a laser based additive manufacturing technology.

The intrinsic feature of laser processing of materials is that, at a certain time, only a small volume of the material is thermally treated, resulting in extremely high-temperature gradients within the sample. Unlike metals, direct laser fabrication of raw lunar regolith is difficult due to 
thermal transient stresses and residual stresses associated with melting/resolidification and the considerably high melting point of ceramics. Thermal stresses that arise from the contraction can cause distortions and possibly failure by delamination or cracking. It is also believed that the raw regolith will require a binder material in order to get the best possible melt during direct part manufacturing in a freeform environment ${ }^{17}$. In spite of these adversities, herein we demonstrate direct fabrication of bulk parts from raw lunar regolith simulant (JSC-1AC) using lasers. The lunar regolith simulant, Johnson Space Center-1AC (JSC-1AC) used in this study was obtained from Orbital Technologies Inc. Madison, WI. JSC-1AC has been designed to be chemically and mineralogically similar to lunar mare regolith ${ }^{15,18}$. JSC-1AC contains almost half silicon oxide with the aluminum oxide, calcium oxide, iron oxide and magnesium oxide comprising the majority of the balance ${ }^{19}$. The primary mineral component is the plagioclase solid solution series. Olivine and pyroxene minerals are also present. The as-received JSC-1AC simulant with particle size $\delta 5 \mathrm{~mm}$ has been screened to a particle size in the range of 50 and $150 \mu \mathrm{m}$ suitable for Laser Engineered Net Shaping (LENS ${ }^{\text {TM}}$ ) equipment.

\section{Results and Discussion}

Initially, a series of experiments were conducted using different laser powers $(50-200 \mathrm{~W})$, scan speeds $\left(10-30 \mathrm{~mm} \mathrm{~s}^{-1}\right)$, and powder feed rates $\left(10-20 \mathrm{~g} \mathrm{~min}^{-1}\right)$ to optimize process parameters. Since the laser absorption of materials is directly proportional to their electrical resistivity, untreated regolith, an insulator, can absorb and retain a large amount of incident laser energy. Therefore, complete melting of regolith powder was observed at a laser power as low as $50 \mathrm{~W}$. Further increase in the laser power beyond $50 \mathrm{~W}$, resulted in severe spreading of liquid regolith during layer-wise deposition due to low melt viscosity. Similar observations were made with decreasing scan speeds and powder feed rates during deposition. The results of our initial rigorous experimentation indicated successful melting and deposition of raw lunar regolith is a function of incident laser energy, which depended on laser power, scan speed and powder feed rate. Dense parts without any macroscopic defects were produced at laser energy levels as a low as $2.12 \mathrm{~J} \mathrm{~mm}^{-2}$, corresponding to a laser power of $50 \mathrm{~W}$, a scan speed of $20 \mathrm{~mm} \mathrm{~s}^{-1}$, and a powder feed rate of $12.36 \mathrm{~g} \mathrm{~min}^{-1}$. Figure 1a shows typical lunar regolith (JSC-1AC) parts fabricated by direct melting of raw regolith powder using Laser Engineered Net Shaping (LENS ${ }^{\mathrm{TM}}$ ). The parts 
exhibited no visible cracks but sequential deposition of material layers produced distinct layered structure along the part axis. The smooth and shiny surface of regolith parts indicated complete melting and resolidification of lunar regolith simulant during laser processing. The bulk density of parts was between $92 \%$ and $95 \%$ of theoretical density of lunar regolith simulant (JSC-1AC).

Figure 1. Typical lunar regolith simulant (JSC-1AC) parts made using LENS ${ }^{\mathrm{TM}}$. High magnification Field Emission Scanning Electron Microscopy (FESEM) image showing nanoscale microstructural features in the LENS ${ }^{\mathrm{TM}}$ processed parts.

Figure 1b shows nano scale microstructure with grain size in the range of $30-100 \mathrm{~nm}$ formed in LENSTM processed JSC-1AC parts. The formation of fine microstructural features in laser processed regolith parts was attributed to the inherent rapid cooling rates, typically between $10^{3}$ and $10^{5} \mathrm{~K} \mathrm{~s}^{-1}$, associated with laser processing. High cooling rates might also form nonequilibrium or amorphous phases during solidification. Since the present regolith simulant (JSC1AC) contains $\sim 50 \%$ silicon oxide, it was expected to form an amorphous phase during laser processing due to its inherently high cooling rates. The X-ray diffraction pattern of LENS ${ }^{\mathrm{TM}}$ processed lunar regolith (JSC-1AC) parts and JSC-1AC feedstock powder is shown in Figure 2. Feedstock lunar regolith simulant showed the presence of crystalline pyroxene, anorthite plagioclase solid solution series, olivine, and ileminite phases. However, it appeared that after laser processing, the regolith lost its crystalline structure and exhibited broad and possibly very weak crystalline peaks. These results suggest that laser processing transformed crystalline regolith in to nanocrystalline and/or amorphous regolith structures. It is hypothesized that laser processing homogenized the JSC-1AC lunar regolith simulant as result of complete melting and resolidification. The regolith parts with extremely fine microstructural features exhibited a high hardness of $500 \pm 18 \mathrm{Hv}$, which was comparable with that of conventional soda lime glass (545 $\pm 20 \mathrm{Hv}$ ). However, it is believed that nanosized microstructural features could potentially enhance the fracture toughness of laser processed regolith parts. 
Figure 2. X-ray diffraction comparing fine and amorphous structures of LENS ${ }^{\mathrm{TM}}$ processed lunar regolith simulant (JSC-1AC) parts to crystalline JSC-1AC powder.

To complement the XRD measurements and to determine possible formation of amorphous phase, the as-received powder and LENS ${ }^{\mathrm{TM}}$ processed samples have been characterized using differential scanning calorimetry (DSC). Figure 3 compares the DSC data of LENS ${ }^{\mathrm{TM}}$ processed sample with that of as-received JSC-1AC powder. Both samples exhibited very small second order endothermic event characteristic of the glass transition $(\mathrm{Tg})$ followed by one exothermic peak associated with crystallization (Tc) and melting between 1120 and $1160^{\circ} \mathrm{C}$. The presence of endo- and exothermic peaks confirmed the presence of small amount of glassy phase in both samples. The crystallization peak of starting feedstock powder shifted to higher temperatures as a result of laser processing. The crystallization occurred between 830 and $930^{\circ} \mathrm{C}$ (peak temperature: $894^{\circ} \mathrm{C}$ ) for laser processed regolith samples, and it was between 750 and $890^{\circ} \mathrm{C}$ (peak temperature: $810^{\circ} \mathrm{C}$ ) for feedstock powder. Present $\mathrm{Tg}$ and Tc temperatures of feedstock lunar regolith simulant (JSC-1AC) were close to the literature values of this material ${ }^{20}$. Another significant difference between the feedstock powder and laser processed regolith was the area under the crystallization peak. Since the area under the crystallization curves is related to the amount of glass content, it can be inferred that the laser processed sample released more exothermic energy than as-received powder. The exothermic energy of regolith increased from $27.85 \mathrm{~J} \mathrm{~g}^{-1}$ to $36.01 \mathrm{~J} \mathrm{~g}^{-1}$ due to laser processing. High magnitude of exothermic energy after laser processing suggests formation of some non-equilibrium/amorphous phases in JSC-1AC lunar regolith simulant.

Formation of glassy/amorphous structure during laser processing depends on achievable cooling rates as a function of laser parameters. In general, lower heat input increases the thermal gradients near the melt zone and consequently high cooling rates can be achieved. Although the local cooling rate is not directly measured in the present work, within the melt zone the cooling rate, $\mathrm{dT} / \mathrm{dt}\left(\mathrm{K} \mathrm{s}^{-1}\right)$, can be expressed as the product of solidification velocity $\mathrm{R}\left(\mathrm{mm} \mathrm{s}^{-1}\right)$ and the local temperature gradient $\mathrm{G}\left(\mathrm{K} \mathrm{mm}^{-1}\right)^{21}$. Experimentally it has been shown that $\mathrm{R}$ is on the order of laser scan speed $(\mathrm{v})^{22}\left(20 \mathrm{~mm} \mathrm{~s}^{-1}\right.$ for the defect free samples made in the present work) and $\mathrm{G}$ 
is on the order of $\sim 100 \mathrm{~K} \mathrm{~mm}^{-123}$. Thus under present experimental conditions, the cooling rate in the melt zone is expected to be on the order of $\sim 2000 \mathrm{~K} \mathrm{~s}^{-1}$, which was responsible for the formation of glassy/amorphous structure in the LENSTM processed parts. However, better estimation of cooling rates should take into consideration of the thermal conductivity of substrate/deposit materials, incident laser energy, temperature variation within the melt zone and substrate or prior deposit temperature.

Figure 3. DSC results showing the crystallization behavior of LENS ${ }^{\mathrm{TM}}$ processed and asreceived lunar regolith simulant (JSC-1AC).

XPS was performed on a Thermo Scientific K-Alpha X-ray spectrometer at a base pressure of 1 x $10^{-9}$ mbar using an Alk $\alpha$ source at an energy of $1486.6 \mathrm{eV}$. The analysis was performed on three specimens from the before and after processing sample sets. The mean relative atomic concentrations of the detected elements in each sample set was summarized in Table 1. From this data, it was observed that the main difference between the before and after LENS prepared samples was an increase in the carbon, aluminum, sodium, and calcium concentrations after processing. Carbon is always present on the surface of any sample that has been exposed to atmosphere, whether due to handling contamination or chemi- or physiabsorbed $\mathrm{CO}$ and $\mathrm{CO} 2$, and has been detected in previous analyses of JSC- $1 \mathrm{~A}^{24}$. The values of the aluminum and sodium concentrations, however, were within the standard deviation of both sample sets. However increases in carbon and calcium concentrations were noticeably larger in the processed samples. Higher resolution scans of the $\mathrm{C} 1 \mathrm{~s}$ and $\mathrm{Ca} 2 \mathrm{p}$ peaks were performed on the before and after processed samples. The peak positions were referenced to the standard carbon C1s peak at 286.5 $\mathrm{eV}^{25}$ and the overlay spectra are shown in Figures 4 and 5. The data summary is shown in Table 2.

Table 1. Relative atomic concentrations derived from the XPS data 


\begin{tabular}{|c|c|c|c|c|c|c|c|c|c|}
\hline Sample & O1s & Mg1s & Si2p & A12p & C1s & Na1s & Fe2p & Ca2p & Ti2p \\
\hline Before & 58.09 & 2.64 & 13.26 & 4.85 & 11.49 & 2.85 & 3.72 & 2.51 & 0.58 \\
\hline After & 52.04 & 2.26 & 11.97 & 5.11 & 19.21 & 3.58 & 2.35 & 3.09 & 0.38 \\
\hline
\end{tabular}

Figure 4. XPS overlay spectra of the C1s peaks for the before (red) and after processed (green) samples

Figure 5. XPS overlay spectra of the Ca2p peaks for the before (red) and after processed (green) samples

Table 2. Peak positions of the $\mathrm{Cls}$ and Ca2p peaks

\begin{tabular}{|c|c|c|}
\hline Sample & C1s & Ca2p \\
\hline Before & $284.6 \mathrm{eV}$ & $347.83 \mathrm{eV}$ \\
\hline After & $284.6 \mathrm{eV}$ & $347.19 \mathrm{eV}$ \\
\hline
\end{tabular}

From Figure 5, there is a shift to lower binding energy of the Ca peak positions, indicating the $\mathrm{Ca}$ has been reduced from a higher state to what corresponds to $\mathrm{CaCO}_{3} \cdot{ }^{26}$ The conclusion is that the carbon in the samples reacted upon processing with the calcium in the plagioclase $\left(\mathrm{NaAlSi}_{3} \mathrm{O}_{8} / \mathrm{CaAl}_{2} \mathrm{Si}_{2} \mathrm{O}_{8}\right)$ that is present in JSC-1A forming $\mathrm{CaCO}_{3}$ that has diffused to the surface of the particles and constitutes the major surface compositional difference between the as-received and processed JSC-1A.

A combination of laser parameters resulting in a $2.12 \mathrm{~J} \mathrm{~mm}^{-2}$ laser energy appeared to be ideal for generating a melt pool necessary for lunar regolith powder deposition without excessive liquid pool spreading and cracking of solidified parts. While current experimental results clearly demonstrated that net shape regolith simulant parts can be fabricated using LENS $^{\mathrm{TM}}$ by exploiting its capabilities, it is still important to carry out further investigations to make larger parts, lunar regolith coatings, and composites reinforced with lunar regolith. Such investigations would 
likely to expand the potential of $\mathrm{LENS}^{\mathrm{TM}}$ as an efficient tool to explore fabrication and repair of parts using unprocessed lunar regolith. Other potential application of laser processing include fabrication of lunar regolith substrates for solar cells ${ }^{8}$, lunar regolith coatings on habitat structures for shielding against space radiation ${ }^{9,10}$, and lunar regolith reinforced metal matrix composites for critical load bearing parts/structures. Finally, further critical evaluation of physical, mechanical and functional properties of laser processed lunar regolith is very important.

\section{Conclusions and Future Work}

Preliminary results described within this report demonstrated the feasibility of manufacturing ceramic parts from lunar simulant through LENS processing. More work will need to be performed to develop more complex articles, such as hinging joints that characterize the interconnected elements of a non-textile mat ${ }^{10}$ as shown in Figure 6, that could be shaped from terrestrial and extraterrestrial mineral compositions. Martian soil simulants will be the focus of a future case study.

\section{Methods}

The lunar regolith simulant used in this study, Johnson Space Center-1AC (JSC-1AC) with particle size $\delta 5 \mathrm{~mm}$, was screened to a particle size in the range of 50 and $150 \mu \mathrm{m}$ suitable for Laser Engineered Net Shaping (LENSTM) equipment. Bulk lunar regolith structures were fabricated using a LENSTM-750 (Optomec, Albuquerque, NM) unit with a $0.5 \mathrm{~kW}$ continuous wave $\mathrm{Nd}-\mathrm{YAG}$ laser on a 3-mm-thick aluminum alloy substrate. LENS $^{\mathrm{TM}}$ processing was carried out in a glove box containing argon atmosphere with oxygen content less than $10 \mathrm{ppm}$. Initially, a series of experiments were conducted using a layer thickness of $254 \mu \mathrm{m}$ at different laser powers, scan speeds, and powder feed rates to optimize the process parameters. Dense parts (8$10 \mathrm{~mm}$, height $25-30 \mathrm{~mm}$ ) without any macroscopic defects were produced at a laser power of 50 $\mathrm{W}$, a scan speed of $20 \mathrm{~mm} \mathrm{~s}^{-1}$, and a powder feed rate of $12.36 \mathrm{~g} \mathrm{~min}^{-1}$. X-ray diffraction (XRD) was carried out to identify the phases using a Siemens D 500 Kristalloflex diffractometer 
(Siemens, Madison, WI) with a step size of 0.05 and a $2 \Theta$ range of $20-60^{\circ}$. Differential scanning calorimetry (DSC) was done using Netzch, STA-409PC thermal analyzer (Netzch, Burlington, MA) at a heating rate of $10^{\circ} \mathrm{C} \mathrm{min}^{-1}$ under flowing air. After being polished, the cross sectional microstructures of LENSTM processed regolith parts were characterized using Field Emission Scanning Electron Microscope (FESEM, FEI-SIRION, Portland, OR). Vickers microhardness measurements were performed on laser processed regolith samples using a $200 \mathrm{~g}$ load and a holding time of $15 \mathrm{~s}$, and the average value of 10 measurements was reported.

Figure 6. Conceptual interlocking ceramic mat ${ }^{10}$.

\section{References}

1. Stanley, D., Cook, S., \& Connolly, J. NASA's Exploration Systems Architecture Study. NASA TM-2005-214062, November 2005.

2. Sammells, A.F. \& Semkow K.W. The Electrochemical Generation of Useful Chemical Species from Lunar Materials. J Power Sources 22, 285-291 (1988).

3. Taylor, L.A. \& Carrier, W.D. Production of Oxygen on the Moon Which Processes are Best and Why. AIAA Journal 30 [12], 2858-2863 (1992).

4. Grugel, R. N. \& Toutanji, H. Sulfur concrete for lunar applications-sublimation concerns, Advances in Space Res. 41, 103-112 (2008).

5. Allen, C. C., Graf, J. C., McKay, D. S. Sintering bricks on the Moon, in: Engineering, Construction, and Operations in Space IV, 1994, pp. 1220-1229.

6. Faierson, E. J., Logan, K. V., Stewart, B. K. \& Hunt, M. P. Demonstration of concept for fabrication of lunar physical assets utilizing lunar regolith simulant and a geothermite reaction. Acta Astronautica doi:10.1016/j.actaastro.2009.12.006 (2010).

7. Taylor. L. A. \& Meek, T. T. Microwave Sintering of Lunar Soil: Properties, Theory, and Practice. J Aerospace Eng. 18, 188-196 (2005). 
8. Horton, C., Gramajo, C., Alemu, A., Williams, L., Ignatiev, A. \& Freundlich, A. First demonstration of photovoltaic diodes on lunar regolith-based substrate. Acta Astronautica 56, $537-545$ (2005).

9. Miller, J., Taylor, L., Zeitlin, C., Heilbronn, L., Guetersloh, S., DiGiuseppe, M., Iwata, Y. \& Murakami, T. Lunar soil as shielding against space radiation. Radiation Measurements 44, 163167 (2009).

10. Roberson, L.B., Hintze, P., \& O'Connor, G.W. A Conceptual Study for the Autonomous Direct Forming of Lunar Regolith into Flexlock ${ }^{\mathrm{TM}}$ Geomats for Lunar Surface Operations. $47^{\text {th }}$ AIAA Aerospace Sciences Meeting Exposition, 5-8 Jan. 2009, Orlando, Florida, USA.

11. Poisl, W.H. \& Fabes, B.D. Refractory materials from lunar resources, in Resources of near earth space, Lewis, J.S., Matthews, M.S. \& Guerrieri, M.L. (eds.), Universit of Arizona Press, 351-365 (1993).

12. Howard, R., Patterson, B., Gilley, S. \& Kennedy, J. Fabrication Technology Options Trade Study. Teledyne Brown Engineering, 480RPT0190, 2004.

13. Hammond, M.S., Good, J.E., Gilley, S.D. \& Howard, R.W. Developing fabrication technologies to provide on demand manufacturing for exploration of the moon and mars. $44^{\text {th }}$ AIAA Aerospace Sciences Meeting and Exhibit, 9-12 Jan. 2006, Reno, NV, USA.

14. Phinney, W. C., Criswell, D., Drexler, E. \& Garmirian, J. Lunar resources and their utilization. Spacer-based Manufacturing from Non-terrestrial Materials, O’Neill, G. K. \& O’leary, D. Eds., Prog. Astronaut. Aeronaut. 57, 91-123 (1977).

15. Heiken, G. H., Vaniman, D. T., French, B. M. \& Schmitt, H. H. Lunar Sourcebook. Cambridge University Press, Cambridge (1991).

16. Blacic, J. D. Mechanical properties of lunar materials under anhydrous, hard vacuum conditions: applications of lunar glass structural components, in: Lunar Bases and Space Activities of the 21st Century. Houston, TX, Lunar and Planetary Institute, edited by W. W. Mendell, 1985, p.487-495. 
17. McLemore, C.A., Darby, J.C., Good, J.E. \& Gilley, S.D. Fabrication Capabilities Utilizing In Situ Materials. AIAA Space 2008 Conference \& Exposition, 9-11 September 2008, San Diego, CA, USA.

18. ASA-MSFC, Characterization Summary of JSC-1A Bulk Lunar Mare Regolith Simulant, B.1 ed: NASA Marshall Space Flight Center, 2007.

19. McKay, D., Carter, J., Boles, W., Allen, C. \& Allton, J. JSC-1, A new lunar soil simulant. Proceedings of Space IV, Engineering, Construction, and Operations, American Society of Civil Engineers, 1994, p. 857.

20. Ray, C.S., Reis, S.T. \& Sen S. Characterization and glass formation of JSC-1 lunar and Martian soil simulants. AIP Conf. Proc. 969(1) (January 2008): 908-916.

21. Bontha, S., Klingbeil, N.W., Kobryn, P.A. \& Fraser, H.L. Thermal process maps for predicting solidification microstructure in laser fabrication of thin-wall structures. $\mathrm{J}$ Mater. Process. Technol. 178, 135-142 (2006).

22. Gaumann, M., Henry, S., Cleton, F., Wagniere, J.D. \& Kurz, W. Epitaxial laser metal forming: analysis of microstructure formation. Mater. Sci. Eng. A 271, 232-241 (1999).

23. Hofmeister, W., Griffith, M., Ensz, M. \& Smugeresky, J. Solidification in direct metal deposition by LENS processing. JOM 53 (9), 30-34 (2001).

24. S. Trigwell, J.G. Captain, E.E. Arens, J.W. Quinn, and C.I. Calle, "The use of tribocharging in the electrostatic beneficiation of lunar simulant", IEEE Trans. Ind. Apps., 45, No. 3, pp. 10601067 (2009).

25. C.D. Wagner, "Handbook of X-ray photoelectron spectroscopy", Perkin-Elmer Corp., Eden Prairie, MN, 1999.

26. Database for surface spectroscopies. http:/Www.lasurface.com. 


\section{Acknowledgements}

The authors acknowledge the financial support of W.M. Keck Foundation to purchase the LENS750 system at WSU.

\section{Authors Contributions}

A.B., L.B.R, G.W.O and V.K.B designed the research and A.B. supervised the project. V.K.B. carried out the experiments. All authors contributed to the interpretation of the results as well as writing the paper.

\section{Additional Information}

The authors declare no competing financial interests. 